\title{
Third-Order Leader-Following Consensus in a Nonlinear Multiagent Network via Impulsive Control
}

\author{
Xiaomei Li, ${ }^{1}$ Zhongjun Ma, $^{1}$ Chunhai Li, ${ }^{1}$ and Jinde $\mathrm{Cao}^{2}$ \\ ${ }^{1}$ School of Mathematics and Computing Science, Guilin University of Electronic Technology, Guilin 541004, China \\ ${ }^{2}$ Department of Mathematics, Southeast University, Nanjing 210096, China \\ Correspondence should be addressed to Zhongjun Ma; mzj1234402@163.com
}

Received 15 June 2013; Accepted 24 August 2013

Academic Editor: Qiankun Song

Copyright (c) 2013 Xiaomei Li et al. This is an open access article distributed under the Creative Commons Attribution License, which permits unrestricted use, distribution, and reproduction in any medium, provided the original work is properly cited.

Many facts indicate that the impulsive control method is a finer method, which is simple, efficient, and low in cost, for achieving consensus. In this paper, based on graph theory, Lyapunov stability theory, and matrix theory, a novel impulsive control protocol is given to realize the consensus of the multiagent network. Numerical simulations are performed to verify the theoretical results.

\section{Introduction}

In the past few years, consensus of multiagent networks has been intensively studied in many fields, such as biological, social, mathematical, and physical sciences ones [1-5]. Generally speaking, consensus refers to designing a system algorithm or protocol such that all agents asymptotically reach an agreement on their states. In particular, leaderfollowing consensus means that there exists a virtual leader which specifies an objective for all agents to follow. Recently, some first-order and second-order leader-following consensus problems were discussed by lots of researchers [6-10], and then some novel system algorithms were given via some different control methods, such as pinning control, delay coupling control, adaptive control, and impulsive control [9-14]. In addition, Qin et al. considered consensus in the second-order multiagent system with communication delay in $[15,16]$. Particularly, some multiagent networks cannot be controlled continuously. At this time, the impulsive control becomes a more desirable alternative. The impulsive control is low in cost and then has been widely applied in many fields, such as information science, system control, life science, communication security, and space techniques [17-19]. In the above senses, the impulsive control is very effective for achieving consensus of a multiagent network.

In some real networks, the connections between part nodes are sometimes a failure, and then the network topology may dynamically change over time. Therefore, it is indispensable to consider the case that the network topology is switching. As much as we know, most of the relevant studies focus on second-order consensus for multiagent networks $[11,12]$. When the agent states are influenced by speeds, positions, and accelerations, it is necessary and significative to research the third-order consensus problem of a multiagent network with switching topology. At present, just few works considered the third-order consensus problem. In [20], adaptive third-order leader-following consensus of a nonlinear multiagent network with perturbations was addressed, without using the impulsive control method. In [11], impulsive consensus problem of second-order multiagent network with switching topologies was investigated, without considering its own dynamics. In this paper, we consider the thirdorder consensus problem in a multiagent network with the aforementioned four characters, that is, leader-following, own dynamics, switching topology, and impulsive control. By using the graph theory, Lyapunov stability theory, and matrix theory, some sufficient conditions are obtained to realize the third-order leader-following consensus.

The rest of this paper is designed as follows. Some necessary preliminaries are stated in Section 2 . The consensus of a multiagent network is discussed in Section 3. Numerical examples are given to verify the theoretical results in Section 4. Finally, in Section 5, conclusions are presented. 


\section{Preliminaries}

2.1. Multiagent Network. Information exchange among agents can be modeled by an interaction graph. Let $G=$ $\{V, E, A\}$ be a weighted diagraph with a node set $V=$ $\{1, \ldots, N\}$, an edge set $E \subseteq V \times V$, and a weight adjacency matrix $A=\left(a_{i j}\right)_{N \times N}$. A directed edge denoted by $(i, j)$ means that $j$ has access to node $i$; that is, node $j$ can receive information from node $i$. The elements of matrix $A$ are defined such that $a_{i j}>0$ for $(j, i) \in E$, while $a_{i j}=0$ for $(j, i) \notin E$. Let $a_{i i}=0$ for $i \in V$. The set $\aleph_{i}=$ $\{j \in V \mid(j, i) \in E\}$ is used as the neighbor set of node $i$. When the communication topology is switching, the neighbor set is time-varying, and then $\aleph_{i}(t)=\left\{j \in V: a_{i j}(t)>0\right\}$. Let

$$
a_{i j}(t)=\left\{\begin{array}{lc}
1, & j \in \aleph_{i}(t) \\
0, & \text { otherwise }
\end{array}\right.
$$

The out-degree of node $i$ is defined by $\operatorname{deg}(i)=\sum_{j=1}^{N} a_{i j}=$ $\sum_{j \in \aleph_{i}} a_{i j}=d_{i}$. If the degree matrix of digraph $G$ is $D=$ $\operatorname{diag}\left(d_{1}, \ldots, d_{N}\right)$, then the Laplacian matrix of digraph $G$ is $L=D-A$.

A directed path from node $i$ to node $j$ in the directed graph $G$ is a sequence of edges $\left(i, j_{1}\right),\left(j_{1}, j_{2}\right), \ldots,\left(j_{l}, j\right)$ with distinct nodes $j_{k}, k=1, \ldots, l$. A digraph $G$ has a directed spanning tree if there exists at least one node called root which has a directed path to all the other nodes.

For a leader-follower multiagent network, suppose that the leader (labeled by 0 ) is denoted by node 0 , and the followers are denoted by the nodes $1,2, \ldots, N$. The graph $\bar{G}$ is consisting of the leader and the followers with communication topology. The connection weight between the $i$ th follower and the leader is represented by $b_{i}, i \in V$. If the $i$ th follower is connected to the leader, then $b_{i}>0$; otherwise, $b_{i}=0$. Let $B=\operatorname{diag}\left\{b_{1}, b_{2}, \ldots, b_{N}\right\}$.

Following, we address the multiagent network with switching topology. The set $\Omega=\left\{\bar{G}_{1}, \bar{G}_{2}, \ldots, \bar{G}_{m}\right\}$ is used as a set of the graphs with all possible topology, which includes all possible graphs (involving $N$ agents and a leader). We define a switching signal $\tau:[0,+\infty) \rightarrow \mathrm{P}=\{1,2, \ldots, m\}$, which determines the topology structure that corresponds to the network. When the topology is switching, the Laplacian matrix $L$ and the matrix $B$ are also switching, which are denoted by $L_{\tau(t)}$ and $B_{\tau(t)}$.

The following assumptions are needed to derive our main results.

Assumption 1 (see [21]). Assume that there exists a constant $\gamma>0$, such that the vector-valued function $f$ satisfies the condition

$$
\begin{aligned}
& (u-w)^{T}(f(t, x, v, u)-f(t, y, z, w)) \\
& \leq \gamma\left((x-y)^{T}(x-y)+(v-z)^{T}(v-z)\right. \\
& \left.\quad+(u-w)^{T}(u-w)\right)
\end{aligned}
$$

for any $x, y, z, u, v, w \in R^{n}$.
2.2. Impulsive Control System. Impulsive control systems can be classified into three types based on the characteristics of plants and control laws [22].

A type-I impulsive control system [22] is given by

$$
\begin{gathered}
\dot{X}=\mathbf{F}(t, X), \quad t \neq \tau_{k}(X), \\
\Delta X=\mathbf{U}(k, Y), \quad t=\tau_{k}(X), \\
Y=\mathbf{G}(t, X),
\end{gathered}
$$

where $X$ and $Y$ are the state variable and the output, respectively. $\mathbf{U}(k, Y)$ is the impulsive control law. In this kind of system, the control input is implemented by the "sudden jumps" of some state variables.

Definition 2 (see [22]). For $t \neq \tau_{k}(X)$, we define the time derivative of the function $V(t, X)$ with respect to system (3) as

$$
\dot{V}(t, X) \triangleq \frac{\partial V(t, X)}{\partial t}+\frac{\partial V(t, X)}{\partial X} \mathbf{F}(t, X)
$$

Type-II and type-III impulsive control systems and more theoretical results are present in [22].

In this paper, a type-I impulsive control system is considered.

\section{Main Results}

Consider that a nonlinear multiagent network consists of $N$ agents with third-order dynamics:

$$
\begin{array}{r}
\dot{x}_{i}(t)=v_{i}(t), \quad \dot{v}_{i}(t)=u_{i}(t), \\
\dot{u}_{i}(t)=f\left(t, x_{i}(t), v_{i}(t), u_{i}(t)\right), \\
i=1,2, \ldots, N,
\end{array}
$$

where $x_{i}=\left(x_{i 1}, \ldots, x_{i n}^{T}\right) \in R^{n}, v_{i}=\left(v_{i 1}, \ldots, v_{i n}\right)^{T} \in R^{n}$, and $u_{i}=\left(u_{i 1}, \ldots, u_{i n}\right)^{T} \in R^{n}$ are the position, velocity, and acceleration states of the $i$ th agent, respectively, and $f=$ $\left(f_{1}, \ldots, f_{n}\right)^{T} \in R^{n}$ is a nonlinear vector-valued continuous function used to describe the self-dynamics of the $i$ th agent.

The virtual leader of the multiagent network (5) is an isolated agent described by

$$
\begin{aligned}
& \dot{x}_{0}(t)=v_{0}(t), \quad \dot{v}_{0}(t)=u_{0}(t), \\
& \dot{u}_{0}(t)=f\left(t, x_{0}(t), v_{0}(t), u_{0}(t)\right),
\end{aligned}
$$

where $x_{0} \in R^{n}, v_{0} \in R^{n}$, and $u_{0} \in R^{n}$ are the position, velocity, and acceleration of the virtual leader, respectively.

For $t \in\left(t_{k-1}, t_{k}\right)$, the state variables $x_{i}(t), v_{i}(t), u(t)$ of the system (5) do dynamically change with ordinary differential equations. And at the moment $t_{k}$, if the $x_{i}(t), v_{i}(t), u(t)$ supervise the impulsive control, then it can result in the jump. Based on the above senses and the impulsive controller of [11] 
and applying impulsive control to (5), we have the following consensus scheme for the multiagent system:

$$
\begin{aligned}
& \dot{x}_{i}(t)=v_{i}(t), \quad\left(t \neq t_{k}\right), \\
& \dot{v}_{i}(t)=u_{i}(t), \quad\left(t \neq t_{k}\right), \\
& \dot{u}_{i}(t)=f\left(t, x_{i}(t), v_{i}(t), u_{i}(t)\right), \quad\left(t \neq t_{k}\right), \\
& \Delta x_{i}\left(t_{k}\right) \triangleq x_{i}\left(t_{k}^{+}\right)-x_{i}\left(t_{k}^{-}\right) \\
& =C_{k}\left(\sum_{j \in \aleph_{i}\left(t_{k}\right)} a_{i j}\left(t_{k}\right)\left(x_{i}\left(t_{k}\right)-x_{j}\left(t_{k}\right)\right)\right. \\
& \left.+b_{i}\left(t_{k}\right)\left(x_{i}\left(t_{k}\right)-x_{0}\left(t_{k}\right)\right)\right) \\
& \Delta v_{i}\left(t_{k}\right) \triangleq v_{i}\left(t_{k}^{+}\right)-v_{i}\left(t_{k}^{-}\right) \\
& =C_{k}\left(\sum_{j \in \aleph_{i}\left(t_{k}\right)} a_{i j}\left(t_{k}\right)\left(v_{i}\left(t_{k}\right)-v_{j}\left(t_{k}\right)\right)\right. \\
& \left.+b_{i}\left(t_{k}\right)\left(v_{i}\left(t_{k}\right)-v_{0}\left(t_{k}\right)\right)\right) \text {, } \\
& \Delta u_{i}\left(t_{k}\right) \triangleq u_{i}\left(t_{k}^{+}\right)-u_{i}\left(t_{k}^{-}\right) \\
& =C_{k}\left(\sum_{j \in \aleph_{i}\left(t_{k}\right)} a_{i j}\left(t_{k}\right)\left(u_{i}\left(t_{k}\right)-u_{j}\left(t_{k}\right)\right)\right. \\
& \left.+b_{i}\left(t_{k}\right)\left(u_{i}\left(t_{k}\right)-u_{0}\left(t_{k}\right)\right)\right) \text {, } \\
& x_{i}\left(t_{0}^{+}\right)=x_{i}\left(t_{0}\right), \quad\left(t_{0} \geq 0\right), \quad x_{i}\left(t_{k}^{-}\right)=x_{i}\left(t_{k}\right), \\
& v_{i}\left(t_{0}^{+}\right)=v_{i}\left(t_{0}\right), \quad\left(t_{0} \geq 0\right), \quad v_{i}\left(t_{k}^{-}\right)=v_{i}\left(t_{k}\right), \\
& u_{i}\left(t_{0}^{+}\right)=u_{i}\left(t_{0}\right), \quad\left(t_{0} \geq 0\right), \quad u_{i}\left(t_{k}^{-}\right)=u_{i}\left(t_{k}\right),
\end{aligned}
$$

where $\Delta x_{i}\left(t_{k}\right), \Delta v_{i}\left(t_{k}\right), \Delta u_{i}\left(t_{k}\right)$ are the jump of the position, velocity, and acceleration of the $i$ th follower agent at the moment $t_{k}$, respectively, $x_{i}\left(t_{k}^{+}\right)=\lim _{h \rightarrow 0^{+}} x_{i}\left(t_{k}+h\right), x_{i}\left(t_{k}^{-}\right)=$ $\lim _{h \rightarrow 0^{-}} x_{i}\left(t_{k}+h\right), v_{i}\left(t_{k}^{+}\right)=\lim _{h \rightarrow 0^{+}} v_{i}\left(t_{k}+h\right), v_{i}\left(t_{k}^{-}\right)=$ $\lim _{h \rightarrow 0^{-}} v_{i}\left(t_{k}+h\right), u_{i}\left(t_{k}^{+}\right)=\lim _{h \rightarrow 0^{+}} u_{i}\left(t_{k}+h\right), u_{i}\left(t_{k}^{-}\right)=$ $\lim _{h \rightarrow 0^{-}} u_{i}\left(t_{k}+h\right), C_{k} \in R^{n \times n}$ is the impulsive controller gain at the moment $t_{k}$, the moments of impulsive satisfy $0 \leq t_{0}<$ $t_{1}<t_{2}<\cdots<t_{k}<t_{k+1}<\cdots$, and $\lim _{k \rightarrow+\infty} t_{k}=+\infty$, $\Delta t_{k}=t_{k}-t_{k-1} \leq \rho<+\infty$ is the impulsive interval, where $k=1,2, \ldots$.

Let $x_{i}(t)-x_{0}(t)=\widehat{x}_{i}(t), v_{i}(t)-x_{0}(t)=\widehat{v}_{i}(t), u_{i}(t)-$ $u_{0}(t)=\widehat{u}_{i}(t), \widehat{x}(t)=\left(\widehat{x}_{1}^{T}(t), \widehat{x}_{2}^{T}(t), \ldots, \widehat{x}_{N}^{T}(t)\right)^{T}, \widehat{v}(t)=$
$\left(\widehat{v}_{1}^{T}(t), \widehat{v}_{2}^{T}(t), \ldots, \widehat{v}_{N}^{T}(t)\right)^{T}, \widehat{u}(t)=\left(\widehat{u}_{1}^{T}(t), \widehat{u}_{2}^{T}(t), \ldots, \widehat{u}_{N}^{T}(t)\right)^{T}$, $e(t)=\left(\widehat{x}^{T}(t), \widehat{v}^{T}(t), \widehat{u}^{T}(t)\right)^{T}$; then the error system with (6) and (7) can be written as

$$
\begin{gathered}
\dot{e}(t)=H e(t)+W, \quad\left(t \neq t_{k}\right), \\
\Delta e\left(t_{k}\right)=\bar{C}_{k} e\left(t_{k}\right), \\
e\left(t_{0}^{+}\right)=e\left(t_{0}\right),
\end{gathered}
$$

where

$$
\begin{gathered}
H=\left(\begin{array}{ccc}
O_{n N \times n N} & I_{n N \times n N} & O_{n N \times n N} \\
O_{n N \times n N} & O_{n N \times n N} & I_{n N \times n N} \\
O_{n N \times n N} & O_{n N \times n N} & O_{n N \times n N}
\end{array}\right), \\
W=\left(\begin{array}{c}
O_{n N \times 1} \\
O_{n N \times 1} \\
F(t, x(t), v(t), u(t))-1_{N} \\
\otimes f\left(t, x_{0}(t), v_{0}(t), u_{0}(t)\right)
\end{array}\right), \\
F(t, x(t), v(t), u(t)) \\
=\left(f^{T}\left(t, x_{1}(t), v_{1}(t), u_{1}(t)\right), \ldots,\right. \\
\left.f^{T}\left(t, x_{N}(t), v_{N}(t), u_{N}(t)\right)\right)^{T}, \\
1_{N}=(1, \ldots, 1)^{T} \in R^{N}, \\
\bar{C}_{k}=\operatorname{diag}\left(\left(L_{\tau(k)}+B_{\tau(k)}\right) \otimes C_{k},\left(L_{\tau(k)}+B_{\tau(k)}\right) \otimes C_{k},\right. \\
\left.\left(L_{\tau(k)}+B_{\tau(k)}\right) \otimes C_{k}\right),
\end{gathered}
$$

$L_{\tau(k)}$ and $B_{\tau(k)}$ are associated with the switching interconnection graph at time $t_{k}, \tau(k) \in \mathrm{P}$.

Definition 3. Denote $e_{x i}(t)=\left\|x_{i}(t)-x_{0}(t)\right\|, e_{v i}(t)=\| v_{i}(t)-$ $v_{0}(t) \|$ and $e_{u i}(t)=\left\|u_{i}(t)-u_{0}(t)\right\|$. The multiagent network (7) with the virtual leader (6) is said to achieve third-order leader-following consensus if the solution of (8) satisfies $\lim _{t \rightarrow+\infty} e_{x i}(t)=0, \lim _{t \rightarrow+\infty} e_{v i}(t)=0$, and $\lim _{t \rightarrow+\infty} e_{u i}(t)=$ $0, i=1,2, \ldots, N$ for any initial condition.

Basing on graph theory, Lyapunov function method, matrix theory, and the proof of Theorem 1 in [11], we have the following theorem.

Theorem 4. Under Assumption 1, if there exists $0<\varphi<1$ such that

$$
\delta_{k} e^{\mu\left(\Delta t_{k}\right)}<\varphi,
$$

where $\delta_{k}$ and $\mu$ are the maximum eigenvalues of matrices

$$
\begin{aligned}
& \left(I_{3 n N \times 3 n N}+\bar{C}_{k}\right)^{T}\left(I_{3 n N \times 3 n N}+\bar{C}_{k}\right), \\
& \left(\begin{array}{ccc}
2 \gamma I_{n N \times n N} & I_{n N \times n N} & O_{n N \times n N} \\
I_{n N \times n N} & 2 \gamma I_{n N \times n N} & I_{n N \times n N} \\
O_{n N \times n N} & I_{n N \times n N} & 2 \gamma I_{n N \times n N}
\end{array}\right),
\end{aligned}
$$

respectively, then the third-order leader-following consensus in the multiagent network (7) is achieved. 
Proof. Consider the following Lyapunov function:

$$
V(e(t))=e^{T}(t) e(t) .
$$

For any $t \in\left(t_{k-1}, t_{k}\right]$, the time derivative of $V(e(t))$ along the trajectory of (8) is

$$
\begin{aligned}
& \mathbf{D} V(e(t))=(\dot{e}(t))^{T} e(t)+e^{T}(t) \dot{e}(t) \\
& =e^{T}(t)\left(H+H^{T}\right) e(t)+2 W^{T} e(t) \\
& =e^{T}(t)\left(H+H^{T}\right) e(t) \\
& +2 \widehat{u}^{T}(t)\left(F(t, x(t), v(t), u(t))-1_{N}\right. \\
& \left.\otimes f\left(t, x_{0}(t), v_{0}(t), u_{0}(t)\right)\right) \\
& =e^{T}(t)\left(H+H^{T}\right) e(t) \\
& +2 \sum_{i=1}^{N} \widehat{u}_{i}^{T}(t)\left(f\left(t, x_{i}(t), v_{i}(t), u_{i}(t)\right)\right. \\
& \left.-f\left(t, x_{0}(t), v_{0}(t), u_{0}(t)\right)\right) \\
& \leq e^{T}(t)\left(H+H^{T}\right) e(t) \\
& +2 \gamma \sum_{i=1}^{N}\left(\widehat{x}_{i}^{T}(t) \widehat{x}_{i}(t)\right. \\
& \left.+\widehat{v}_{i}^{T}(t) \widehat{v}_{i}(t)+\widehat{u}_{i}^{T}(t) \widehat{u}_{i}(t)\right) \\
& =e^{T}(t)\left(H+H^{T}\right) e(t) \\
& +e^{T}(t)\left(\begin{array}{ccc}
2 \gamma I_{n N \times n N} & 0_{n N \times n N} & 0_{n N \times n N} \\
0_{n N \times n N} & 2 \gamma I_{n N \times n N} & 0_{n N \times n N} \\
0_{n N \times n N} & 0_{n N \times n N} & 2 \gamma I_{n N \times n N}
\end{array}\right) e(t) \\
& =e^{T}(t)\left(\begin{array}{ccc}
2 \gamma I_{n N \times n N} & I_{n N \times n N} & 0_{n N \times n N} \\
I_{n N \times n N} & 2 \gamma I_{n N \times n N} & I_{n N \times n N} \\
0_{n N \times n N} & I_{n N \times n N} & 2 \gamma I_{n N \times n N}
\end{array}\right) e(t) \\
& \leq \mu V(e(t)) \text {. }
\end{aligned}
$$

Moreover, for any $t \in\left(t_{k-1}, t_{k}\right]$,

$$
0 \leq V(e(t)) \leq V\left(t_{k-1}^{+}\right) e^{\mu\left(t-t_{k-1}\right)} .
$$
have

For $t=t_{k}^{+}$, similar to the proof of Theorem 1 in [11], we

$$
\begin{aligned}
V\left(e\left(t_{k}^{+}\right)\right) & =e^{T}\left(t_{k}^{+}\right) e\left(t_{k}^{+}\right) \\
& =\left(e\left(t_{k}\right)+\Delta e\left(t_{k}\right)\right)^{T}\left(e\left(t_{k}\right)+\Delta e\left(t_{k}\right)\right) \\
& =e^{T}\left(t_{k}\right)\left(I_{3 n N \times 3 n N}+\bar{C}_{k}\right)^{T}\left(I_{3 n N \times 3 n N}+\bar{C}_{k}\right) e\left(t_{k}\right) ;
\end{aligned}
$$

that is,

$$
V\left(e\left(t_{k}^{+}\right)\right) \leq \delta_{k} V\left(e\left(t_{k}\right)\right)
$$

Consequently, according to (10) and (15), we obtain

$$
V\left(e\left(t_{k}^{+}\right)\right) \leq \delta_{k} V\left(t_{k-1}^{+}\right) e^{\mu\left(t_{k}-t_{k-1}\right)} \leq \varphi V\left(t_{k-1}^{+}\right) .
$$

By recurrence, we have

$$
V\left(e\left(t_{k}^{+}\right)\right) \leq \varphi^{k} V\left(t_{0}\right)
$$

Then,

$$
\lim _{k \rightarrow+\infty} V\left(e\left(t_{k}^{+}\right)\right)=0 .
$$

Using $\Delta t_{k}=t_{k}-t_{k-1} \leq \rho<+\infty$ and (15),

$$
\lim _{t \rightarrow+\infty} V(e(t))=0
$$

that is,

$$
\lim _{t \rightarrow+\infty} e(t)=0 \text {. }
$$

Therefore, third-order leader-following consensus in the multiagent network (5) is achieved under the impulsive controllers (7).

Remark 5. According to the proof of Theorem 4, it is not necessary that all the graphs $G_{1}, G_{2}, \ldots, G_{m}$ have directed spanning tree.

On the basis of Gersgorin disk theorem [23], we get $\mu \leq$ $2(\gamma+1)$. For $\left(I_{3 n N \times 3 n N}+\bar{C}_{k}\right)^{T}\left(I_{3 n N \times 3 n N}+\bar{C}_{k}\right)=I_{3 \times 3} \otimes\left[\left(I_{n N \times n N}+\right.\right.$ $\left.\left.\left(L_{\tau(k)}+B_{\tau(k)}\right) \otimes C_{k}\right)^{T}\left(I_{n N \times n N}+\left(L_{\tau(k)}+B_{\tau(k)}\right) \otimes C_{k}\right)\right]$, accordingly, $\delta_{k}$ is equal to the maximum eigenvalue of $\left(I_{n N \times n N}+\left(L_{\tau(k)}+\right.\right.$ $\left.\left.B_{\tau(k)}\right) \otimes C_{k}\right)^{T}\left(I_{n N \times n N}+\left(L_{\tau(k)}+B_{\tau(k)}\right) \otimes C_{k}\right)$. Therefore, we have the following Corollary 6 (recalling $\Delta t_{k}=t_{k}-t_{k-1} \leq \rho$ and $\mathrm{P}=\{1,2, \ldots, m\})$.

Corollary 6. Under Assumption 1, if there exists $0<\varphi<1$ such that

$$
\delta e^{2(\gamma+1) \rho}<\varphi,
$$

where $\delta=\sup \left\{\delta_{k}\right\}, \delta_{k}$ is the maximum eigenvalue of the matrix $\left(I_{n N \times n N}+\left(L_{\tau(k)}+B_{\tau(k)}\right) \otimes C_{k}\right)^{T}\left(I_{n N \times n N}+\left(L_{\tau(k)}+B_{\tau(k)}\right) \otimes\right.$ $\left.C_{k}\right)$; then, the third-order leader-following consensus in the multiagent network (7) is achieved.

\section{Numerical Simulations}

In this section, we give some numerical examples to verify the theory results given in the previous section.

Consider the following nonlinear function $f$ for multiagent network:

$$
f\left(x_{i}(t), v_{i}(t), a_{i}(t)\right)=\left(\begin{array}{c}
2 a_{i 2}+\left(\left|a_{i 1}+1\right|-\left|a_{i 1}-1\right|\right) \\
a_{i 1}-a_{i 2}+a_{i 3} \\
-2 \cos x_{i 1}+2 \cos v_{i 1}-a_{i 2}-a_{i 3}
\end{array}\right) .
$$

It is easy to verify that the nonlinear function $f$ in (24) satisfies Assumption 1. (Let $\gamma=5$ ).

Following, we consider that the topology of the multiagent network is switching $(\mathrm{P}=\{1,2\})$. 


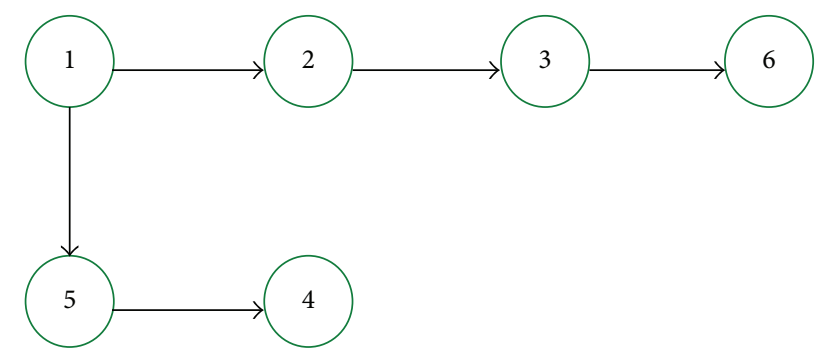

FIGURE 1: Spanning tree of graph $G_{1}$.

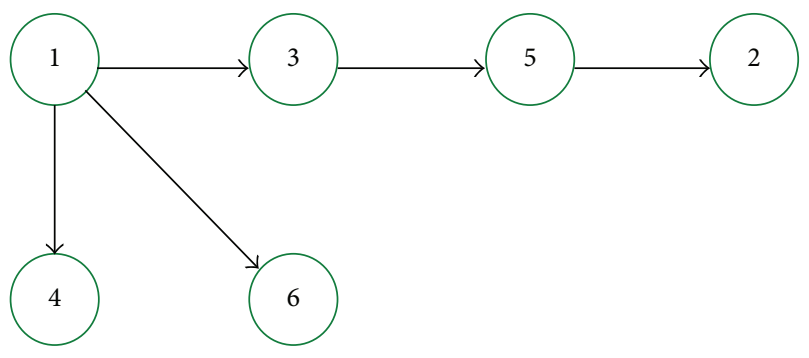

Figure 2: Spanning tree of graph $G_{2}$.

Example 7. Assume that the graphs $G_{1}, G_{2}$ have directed spanning trees. The directed spanning trees of $G_{1}, G_{2}$ are described in Figures 1 and 2. The corresponding matrices of the graphs $G_{1}, \bar{G}_{1}, G_{2}, \bar{G}_{2}$ are $A_{1}, B_{1}, A_{2}, B_{2}$, respectively, where

$$
\begin{gathered}
A_{1}=\left(\begin{array}{llllll}
0 & 0 & 1 & 1 & 0 & 0 \\
1 & 0 & 1 & 0 & 0 & 0 \\
0 & 1 & 0 & 0 & 0 & 1 \\
1 & 0 & 0 & 0 & 1 & 0 \\
1 & 0 & 0 & 0 & 0 & 0 \\
0 & 0 & 1 & 0 & 1 & 0
\end{array}\right), \\
A_{2}=\left(\begin{array}{llllll}
0 & 0 & 0 & 1 & 0 & 1 \\
1 & 0 & 0 & 0 & 1 & 0 \\
1 & 1 & 0 & 0 & 0 & 0 \\
1 & 0 & 0 & 0 & 1 & 0 \\
0 & 1 & 1 & 0 & 0 & 0 \\
1 & 0 & 1 & 0 & 0 & 0
\end{array}\right), \\
B_{1}=\operatorname{diag}(0,1,0,1,0,0), \\
0
\end{gathered}
$$

In time intervals $\left[t_{4 k-1}, t_{4 k}\right)$, the corresponding matrices are $A_{2}, B_{2}, k=1,2, \ldots$ In other time intervals, the corresponding matrices are $A_{1}, B_{1}$. We choose the impulsive gains $C_{k 1}, C_{k 2}$ with topology $\bar{G}_{1}$ and $\bar{G}_{2}$, respectively, as follows:

$$
\begin{aligned}
C_{k 1} & =\left(\begin{array}{ccc}
-0.04 & 0 & 0 \\
0 & -0.04 & 0 \\
0 & 0 & -0.04
\end{array}\right), \\
C_{k 2} & =\left(\begin{array}{ccc}
-0.05 & 0 & 0 \\
0 & -0.05 & 0 \\
0 & 0 & -0.05
\end{array}\right) .
\end{aligned}
$$

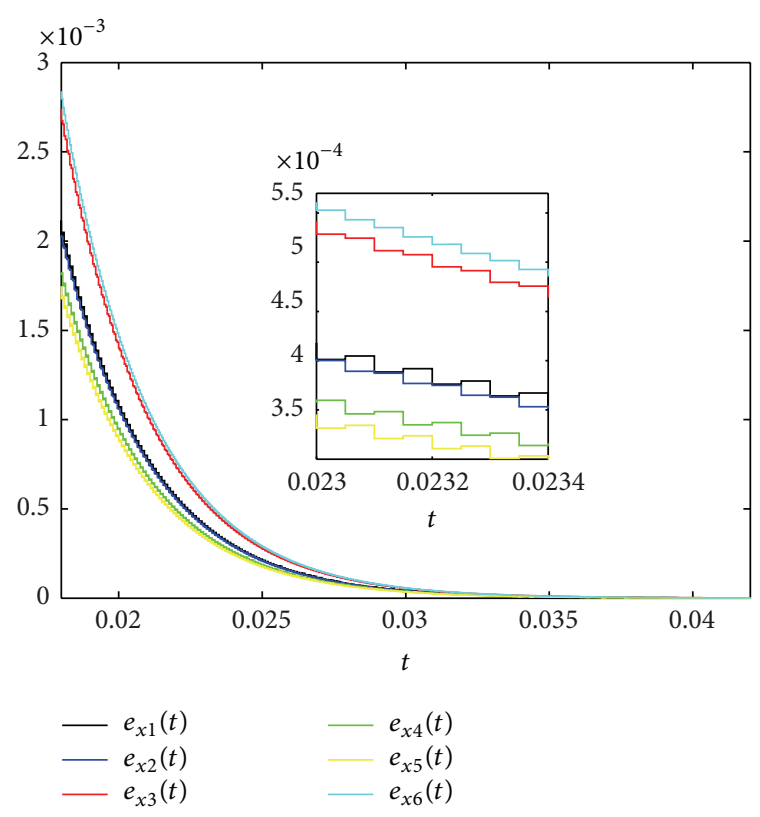

FIgure 3: Position errors $e_{x i}(t)$ of system (7) with a directed spanning tree.

Let the equidistant impulsive interval $\Delta t_{k}=t_{k}-t_{k-1}=$ 0.00005 . Then, $\rho=0.00005$. There exists $\varphi=0.9995$, which satisfies the inequality (23) of Corollary 6. From Figures 3, 4, and 5 , we can see that the position errors $e_{x i}(t)$, the velocity errors $e_{v i}(t)$, and the acceleration errors $e_{a i}(t)$ converge to zero quickly.

Example 8. Assume that the graphs $G_{1}, G_{2}$ do not contain directed spanning trees. Let the corresponding matrices of the graphs $G_{3}, \bar{G}_{3}, G_{4}, \bar{G}_{4}$ be $A_{3}, B_{3}, A_{4}, B_{4}$, respectively, where

$$
\begin{gathered}
A_{3}=\left(\begin{array}{llllll}
0 & 1 & 0 & 0 & 0 & 0 \\
0 & 0 & 0 & 0 & 0 & 0 \\
0 & 0 & 0 & 0 & 0 & 0 \\
1 & 0 & 1 & 0 & 0 & 0 \\
0 & 1 & 1 & 0 & 0 & 0 \\
0 & 0 & 0 & 1 & 1 & 0
\end{array}\right), \\
A_{4}=\left(\begin{array}{llllll}
0 & 0 & 1 & 0 & 0 & 0 \\
1 & 0 & 0 & 1 & 0 & 0 \\
0 & 1 & 0 & 1 & 0 & 0 \\
0 & 0 & 0 & 0 & 0 & 0 \\
0 & 0 & 0 & 0 & 0 & 0 \\
1 & 0 & 0 & 0 & 1 & 0
\end{array}\right), \\
B_{3}=\operatorname{diag}(0,1,1,0,0,0), \\
0
\end{gathered}
$$

Similar to Example 7, in time intervals $\left[t_{4 k-1}, t_{4 k}\right)$, the corresponding matrices are $A_{4}, B_{4}, k=1,2, \ldots$, while in other time intervals, the corresponding matrices are $A_{3}, B_{3}$. 


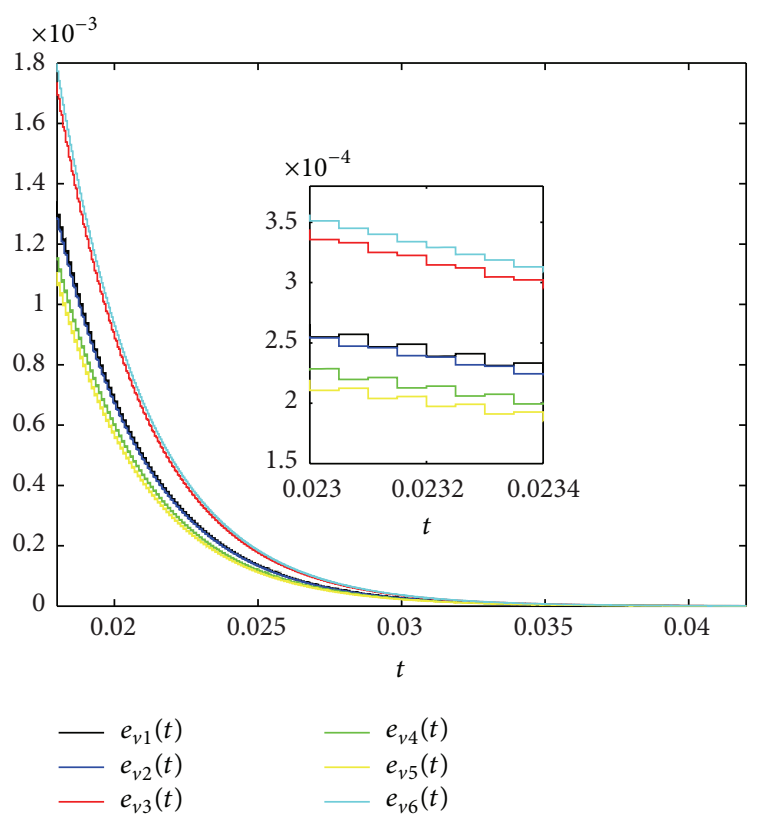

FIgURE 4: Velocity errors $e_{v i}(t)$ of system (7) with a directed spanning tree.

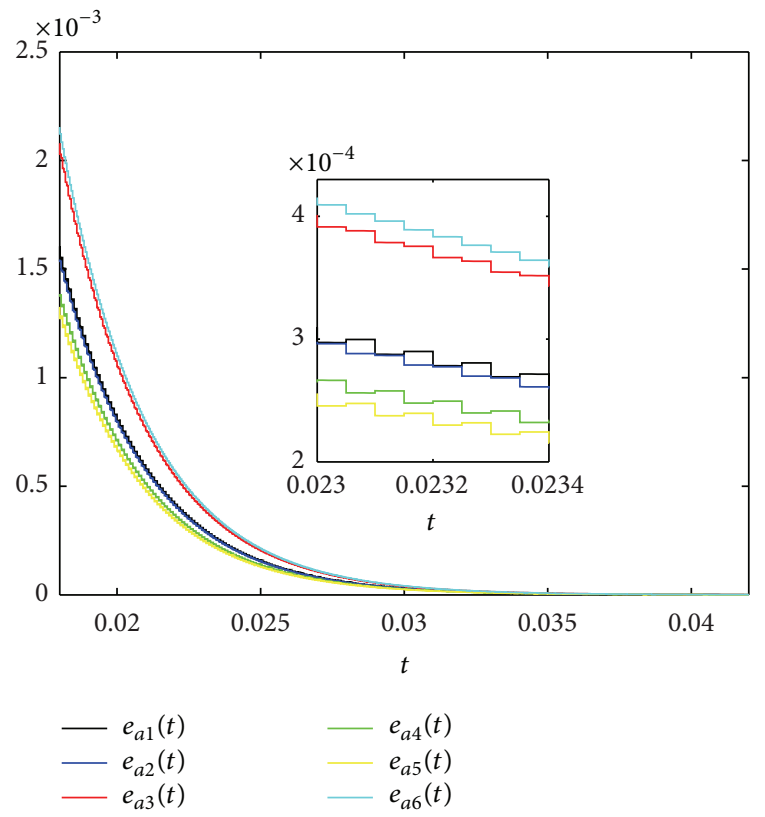

FIgURE 5: Acceleration errors $e_{a i}(t)$ of system (7) with a directed spanning tree.

We choose the impulsive gain $C_{k 3}, C_{k 4}$ with topology $\bar{G}_{3}$ and $\bar{G}_{4}$, respectively, as follows:

$$
\begin{aligned}
C_{k 3} & =\left(\begin{array}{ccc}
-0.07 & 0 & 0 \\
0 & -0.07 & 0 \\
0 & 0 & -0.07
\end{array}\right), \\
C_{k 4} & =\left(\begin{array}{ccc}
-0.08 & 0 & 0 \\
0 & -0.08 & 0 \\
0 & 0 & -0.08
\end{array}\right) .
\end{aligned}
$$

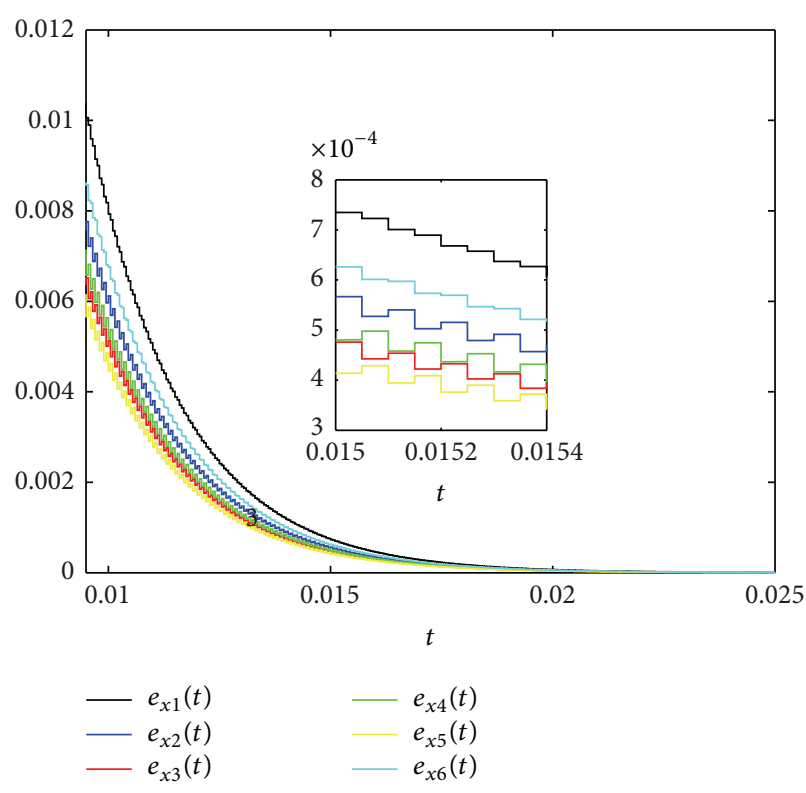

FIgure 6: Position errors $e_{x i}(t)$ of system (7) without a directed spanning tree.

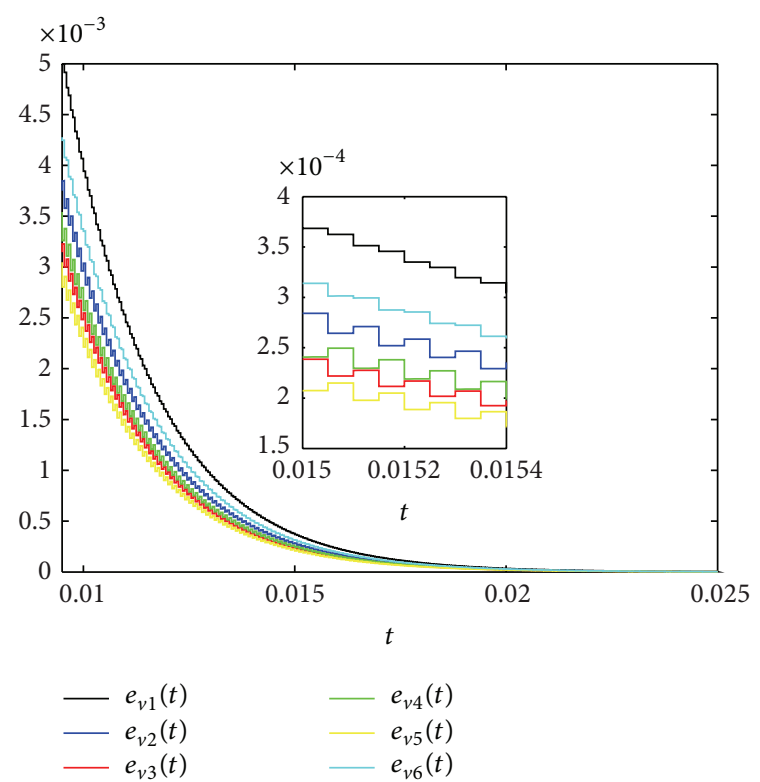

FIGURE 7: Velocity errors $e_{v i}(t)$ of system (7) without a directed spanning tree.

Let the equidistant impulsive interval $\Delta t_{k}=t_{k}-t_{k-1}=$ 0.00005 . Then, $\rho=0.00005$. By some calculations, we can know that there exists $\varphi=0.9995$, which satisfies the inequality (23) of Corollary 6. From Figures 6, 7, and 8, we can see that the position errors $e_{x i}(t)$, the velocity errors $e_{v i}(t)$, and the acceleration errors $e_{a i}(t)$ converge to zero quickly. It shows that the condition on a directed spanning tree is not necessary to realize consensus of the multiagent network (7) under the impulsive control. In addition, the researchers considered the second-order multiagent system 


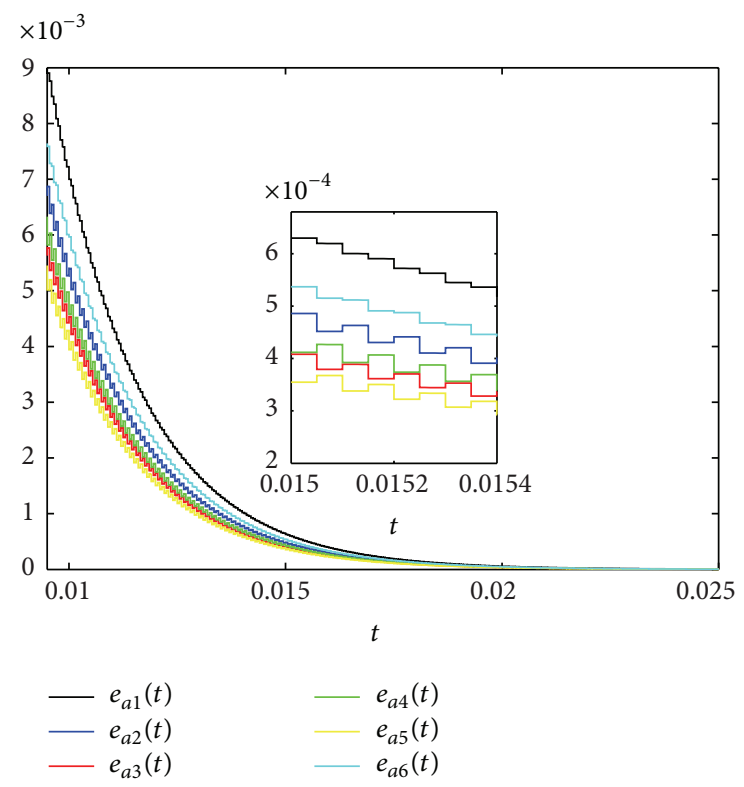

Figure 8: Acceleration errors $e_{a i}(t)$ of system (7) without a directed spanning tree.

with communication delay in $[21,22]$. In our future work, we will study the third-order consensus problem for the multiagent systems with communication delay.

\section{Conclusion}

By using graph theory, Lyapunov stability theory, and matrix theory, third-order leader-following consensus problem of a nonlinear multiagent network is studied in this paper. By designing proper impulsive controllers, a new criterion on realizing consensus in the multiagent network with switching topology is achieved. Finally, numerical simulations are provided to illustrate the theoretical results. In our future work, we will study the third-order consensus problem for the multiagent systems with communication delay.

\section{Acknowledgments}

This work was supported by the National Natural Science Foundation of China (nos. 60964006, 61004101, and 11162004) and the Science Foundation of Guangxi Province, China (no. 2013GXNSFAA019006).

\section{References}

[1] T. Vicsek, A. Czirok, E. B. Jacob, I. Cohen, and O. Schocher, "Novel type of phase transition in a system of self-driven particles," Physical Review Letters, vol. 75, pp. 1226-1229, 1995.

[2] A. Jadbabaie, J. Lin, and A. S. Morse, "Coordination of groups of mobile autonomous agents using nearest neighbor rules," IEEE Transactions on Automatic Control, vol. 48, no. 6, pp. 988-1001, 2003.

[3] J. Q. Lu, D. Ho, and J. Kurths, "Consensus over directed static networks with arbitrary finite communication delays," Physical Review E, vol. 80, no. 6, Article ID 066121, 7 pages, 2009.
[4] J. H. Qin, W. X. Zheng, and H. J. Gao, "Consensus of multiple second-order vehicles with a time-varying reference signal under directed topology," Automatica, vol. 47, no. 9, pp. 19831991, 2011.

[5] R. Olfati-Saber and R. M. Murray, "Consensus problems in networks of agents with switching topology and time-delays," IEEE Transactions on Automatic Control, vol. 49, no. 9, pp. 15201533, 2004.

[6] K. Peng and Y. P. Yang, "Leader-following consensus problem with a varying-velocity leader and time-varying delays," Physica A, vol. 388, pp. 193-208, 2009.

[7] C. Q. Ma, T. Li, and J. F. Zhang, "Consensus control for leaderfollowing multi-agent systems with measurement noises," Journal of Systems Science \& Complexity, vol. 23, no. 1, pp. 35-49, 2010.

[8] W. W. Yu, G. R. Chen, and M. Cao, "Distributed leader-follower flocking control for multi-agent dynamical systems with timevarying velocities," Systems \& Control Letters, vol. 59, no. 9, pp. 543-552, 2010.

[9] Q. Song, J. D. Cao, and W. W. Yu, "Second-order leaderfollowing consensus of nonlinear multi-agent systems via pinning control," Systems \& Control Letters, vol. 59, no. 9, pp. 553$562,2010$.

[10] J. P. Hu and Y. G. Hong, "Leader-following coordination of multi-agent systems with coupling time delays," Physica A, vol. 374, no. 2, pp. 853-863, 2007.

[11] Q. Zhang, S. H. Chen, and C. C. Yu, "Impulsive consensus problem of second-order multi-agent systems with switching topologies," Communications in Nonlinear Science and Numerical Simulation, vol. 17, no. 1, pp. 9-16, 2012.

[12] H. B. Jiang, Q. S. Bi, and S. Zheng, "Impulsive consensus in directed networks of identical nonlinear oscillators with switching topologies," Communications in Nonlinear Science and Numerical Simulation, vol. 17, no. 1, pp. 378-387, 2012.

[13] H. B. Jiang, J. J. Yu, and C. G. Zhou, "Consensus of multiagent linear dynamic systems via impulsive control protocols," International Journal of Systems Science, vol. 42, no. 6, pp. 967976, 2011.

[14] X. Han and J.-A. Lu, "Impulsive control induced effects on dynamics of single and coupled ODE systems," Nonlinear Dynamics, vol. 59, no. 1-2, pp. 101-111, 2010.

[15] J. H. Qin and H. J. Gao, "A sufficient condition for convergence of sampled-data consensus for double-integrator dynamics with nonuniform and time-varying communication delays," IEEE Transactions on Automatic Control, vol. 57, no. 9, pp. 2417-2422, 2012.

[16] J. Qin, H. Gao, and W. X. Zheng, "Second-order consensus for multi-agent systems with switching topology and communication delay," Systems \& Control Letters, vol. 60, no. 6, pp. 390-397, 2011.

[17] Z. C. Yang and D. Y. Xu, "Stability analysis and design of impulsive control systems with time delay," IEEE Transactions on Automatic Control, vol. 52, no. 8, pp. 1448-1454, 2007.

[18] H. B. Jiang, J. J. Yu, and C. G. Zhou, "Robust fuzzy control of nonlinear fuzzy impulsive systems with time-varying delay," IET Control Theory \& Applications, vol. 2, no. 8, pp. 654-661, 2008.

[19] Y. G. Sun, L. Wang, and G. M. Xie, "Average consensus in networks of dynamic agents with switching topologies and multiple time-varying delays," Systems \& Control Letters, vol. 57, no. 2, pp. 175-183, 2008. 
[20] M. Sun, Y. Chen, L. Cao, and X. F. Wang, "Adaptive third-order leader following consensus of nonlinear multi-agent systems with perturbations," Chinese Physics Letters, vol. 29, no. 2, Article ID 020503, 2012.

[21] C. Hu, J. Yu, H. J. Jiang, and Z. D. Teng, "Synchronization of complex community networks with nonidentical nodes and adaptive coupling strength," Physics Letters A, vol. 375, no. 5, pp. 873-879, 2011.

[22] T. Yang, Impulsive Control Theory, Springer, New York, NY, USA, 2001.

[23] R. Bhatia, Matrix Analysis, Springer, New York, NY, USA, 1997. 


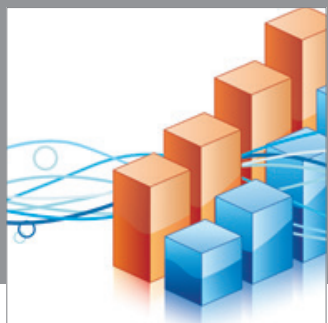

Advances in

Operations Research

mansans

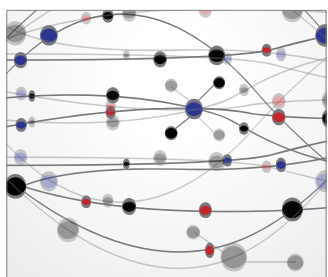

The Scientific World Journal
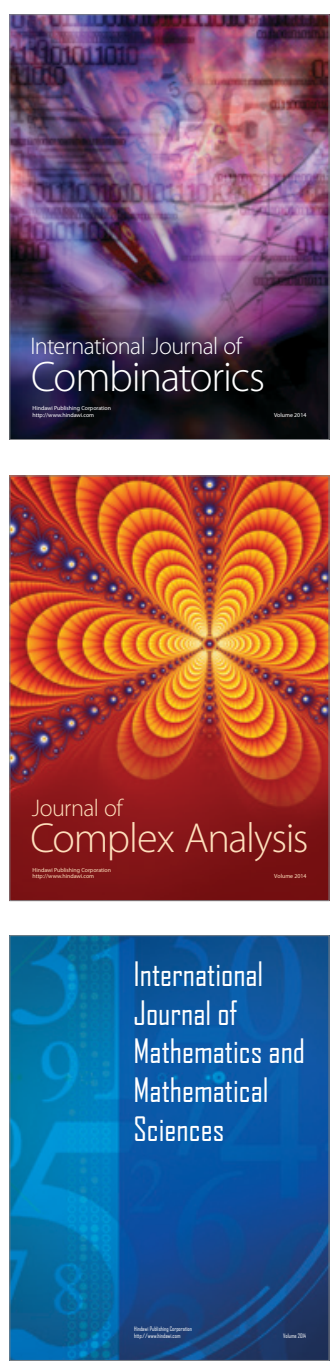
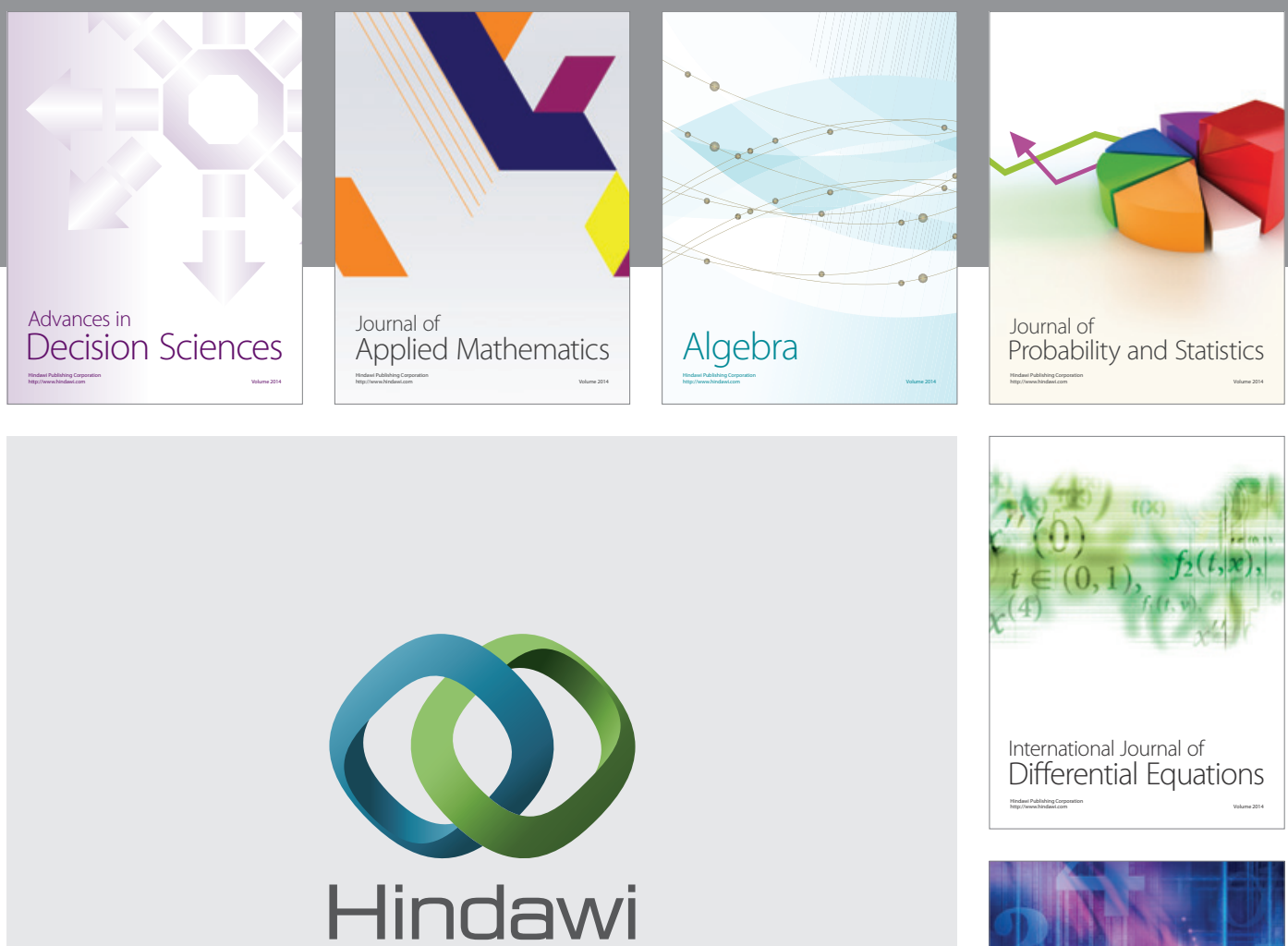

Submit your manuscripts at http://www.hindawi.com
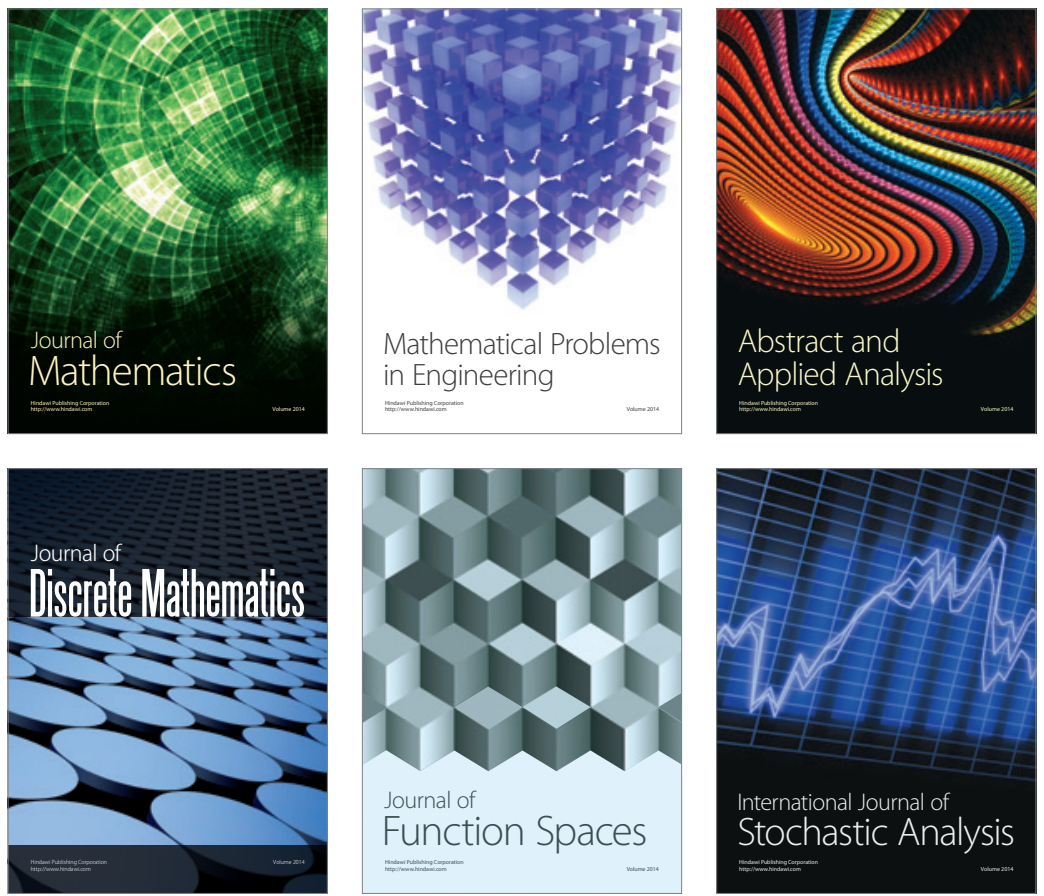

Journal of

Function Spaces

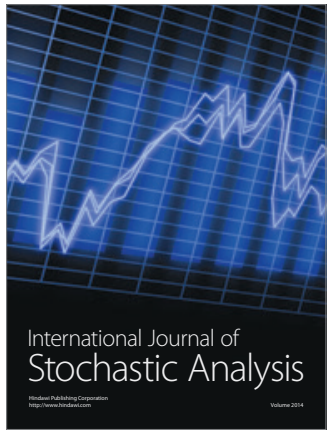

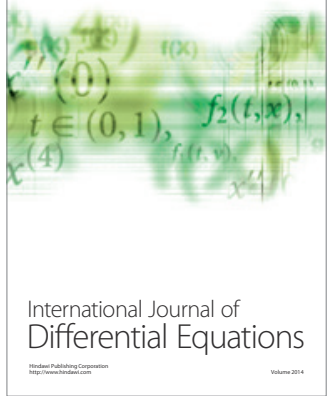
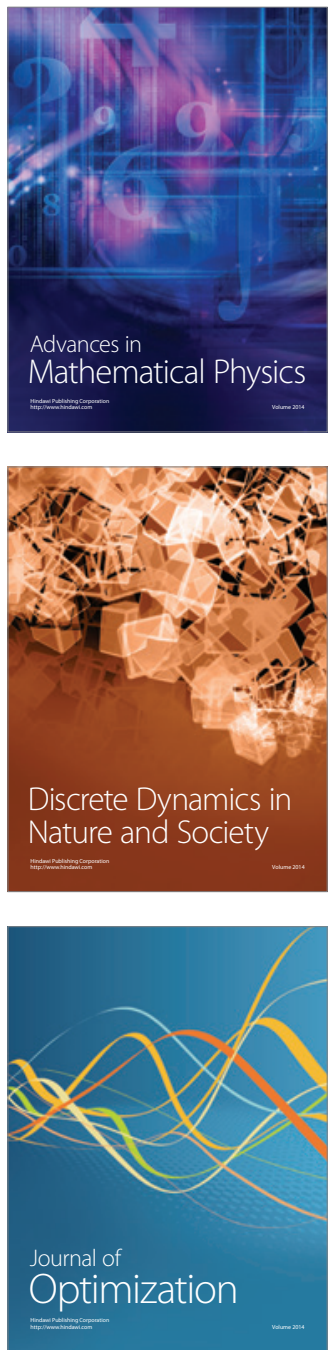